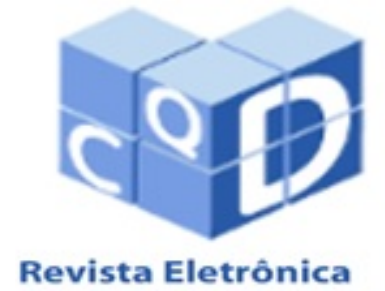

Paulista de Matemática

ISSN 2316-9664

Volume 11, dez. 2017

Edição Iniciação Científica

Anderson Ferreira Sepulveda Universidade de São Paulo Instituto de Física anderson.sepulveda@usp.br

\section{Modelo de Hodgkin-Huxley resolvido pelo método de Runge-Kutta}

Hodgkin-Huxley model solved by Runge-Kutta method

\section{Resumo}

Em 1952, Alan Hodgkin e Andrew Huxley propuseram em um artigo como o potencial de ação é propagado ao longo do axônio de lula gigante. O modelo de Hodgkin-Huxley descreve matematicamente, a partir de conceitos físicos clássicos do Eletromagnetismo, como a membrana e os canais iônicos podem ser vistos como elementos de um circuito elétrico. Como os modelos propostos são equações diferenciais ordinárias, as soluções dessas equações podem ser complicadas de se resolver. Existem ferramentas matemáticas que ajudam a resolver essas equações. Nesse trabalho proponho resolver o modelo de Hodgkin-Huxley através do método de Runge-Kutta. Esse método tem a vantagem de possuir erro menor que o método de Euler. Com a implementação do modelo por esse método, foi obtido valores próximos da solução exata, porém o custo computacional torna cada vez mais difícil de chegar à essa solução.

Palavras-chave: Neurofísica. Modelagem matemática. Equações Diferenciais Ordinárias.

\begin{abstract}
In 1952 Alan Hodgkin and Andrew Huxley proposed in an article how the action potential is propagated along the giant squid axon. The Hodgkin-Huxley model describes mathematically, from classical physical concepts of electromagnetism, how the membrane and ion channels can be seen as elements of an electric circuit. Since the proposed models are ordinary differential equations, the solutions of these equations can be complicated to solve. There are mathematical tools that help solve these equations. In this work I propose to solve the Hodgkin-Huxley model through the Runge-Kutta method. This method has the advantage of having less error than the Euler method. With the implementation of the model by this method, values close to the exact solution were obtained, but the computational cost makes it increasingly difficult to reach this solution.
\end{abstract}

Keywords: Neurophysics. Mathematical Modeling. Ordinary Differential Equations. 


\section{Introdução}

As células nervosas possuem a capacidade de gerar impulsos nervosos que são transmitidos em saltos ao longo de suas membranas e depois transmitir esses impulsos às outras células. Essa capacidade é possibilitada pelas diferenças de concentrações de íons e moléculas orgânicas, que por difusão ou através da bomba de $\mathrm{Na}^{+} / \mathrm{K}^{+}$são trocadas entre o meio intra e extracelular. Existe maior concentração de $\mathrm{Na}^{+}$fora da célula, e as concentrações de $\mathrm{K}^{+}$e $\mathrm{Cl}^{-}$são maiores no meio intracelular. A membrana plasmática, formada por uma bicamada lipídica, possui resistência à condução elétrica. No entanto, a resistência (ou a sua inversa, condutância) é afetada pela presença de canais iônicos. A capacidade desses canais de permitir a passagem de íons através da membrana pode ser modificada por fatores como o potencial da membrana (canais voltagem-dependentes), a concentração de vários mensageiros intracelulares (como canais $\mathrm{Ca}^{2+}$-dependentes), a concentração extracelular de neurotransmissores (canais sinápticos), e alguns canais (ou bombas) são dependentes de energia para manter as concentrações de íons dentro e fora da célula.

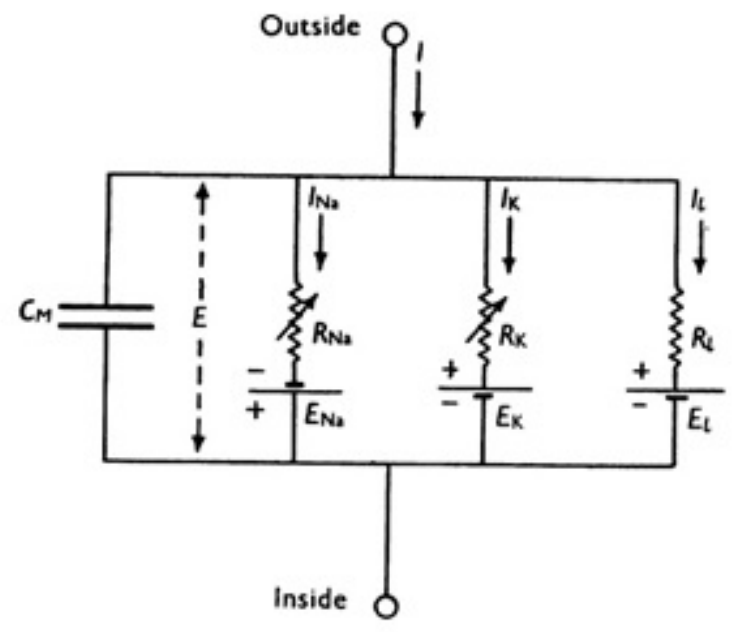

Figura 1: Circuito proposto por Hodgkin e Huxley que representa a membrana celular. $R_{N a}=$ $1 / g_{N a}, R_{K}=1 / g_{K}, R_{L}=1 / \bar{g}_{L} . R_{N a}$ e $R_{K}$ variam com o tempo e com o potencial da membrana. Outros componentes são constantes de Hodgkin e Huxley (1952).

Em 1938, Kenneth Cole e Howard Curtis registraram a atividade no axônio gigante da lula verificando que a condutância da membrana aos íons aumenta de forma muito acentuada durante o potencial de ação (PA). Isso significa que o PA resultaria do movimento dos íons através dos canais. Uma década depois, Alan Hodgkin e Bernard Katz verificaram que a amplitude do PA era reduzida quando a concentração de externa $\mathrm{Na}^{+}$era diminuída. Hodgkin e Katz propuseram que a despolarização que dá início a um potencial de ação faria com que a membrana aumentasse a permeabilidade ao sódio $\left(\mathrm{P}_{\mathrm{Na}^{+}}\right)$por tempo muito curto. Esse influxo sobrepujaria a permeabilidade primária ao $K^{+}$da célula em repouso. Seus dados também sugeriam que a fase descendente do potencial fosse causada por aumento tardio da permeabilidade ao potássio $\left(P_{K}\right)$.

Para testar essa hipótese, era necessário que o potencial da membrana variasse, de forma sistemática, medindo-se, então, as alterações resultantes da condutância da membrana aos íons sódio e potássio, resultantes da abertura e do fechamento dos canais voltagem-dependentes. Experimentalmente, isso é difícil de ser realizado, devido à forte interdependência do potencial de 
membrana e os canais de $\mathrm{Na}^{+}$e $\mathrm{K}^{+}$. Por exemplo, se a membrana for despolarizada o suficiente para abrir alguns dos canais voltagem-dependentes de $\mathrm{Na}^{+}$, ocorre corrente de influxo de íons sódio por esses canais, produzindo mais despolarização.

Essa despolarização adicional faz com que mais canais de $\mathrm{Na}^{+}$se abram, provocando mais corrente de influxo de sódio. Como resultado, é desencadeado um ciclo regenerativo que torna impossível a obtenção de um potencial de membrana estável. Esse ciclo de feedback positivo provoca, eventualmente, o potencial de membrana até o valor do pico do PA. Dificuldade técnica semelhante impede o estudo dos canais ativos de $K^{+}$, responsáveis pela fase decrescente do PA. Em 1949, Cole projetou um aparelho, conhecido como fixador de voltagem (voltage clamp), para resolver esse problema. Pelo uso da técnica de fixação de voltagem no axônio gigante de lula, Hodgkin e Huxley obtiveram no início dos anos 1950 a primeira descrição completa dos mecanismos iônicos responsável pelo PA.

Por convenção, o potencial do meio extracelular é definido como zero. Quando um neurônio está inativo, o excesso de cargas negativas faz com que o potencial dentro da célula seja negativo. Porém, o potencial muda quando o balanço de concentrações é alterado com a abertura e o fechamento dos canais. Em condições normais, o potencial da membrana varia entre $-90 \mathrm{mV}$ e +50 $m V$. Porém, os potenciais medidos dentro do neurônio podem ter valores diferentes, isso porque existem diferentes concentrações iônicas em diferentes partes da célula.

Por causa do excesso de cargas negativas no lado interno e de cargas positivas no lado externo do neurônio, a membrana cria uma capacitância $C_{m}$, a voltagem $V$ através da membrana e um excesso de carga $Q$, que são relacionados à equação do capacitor $Q=C_{m} V$. A capacitância específica da membrana é a capacitância por unidade de área da membrana e é aproximadamente a mesma para todos os neurônios $\left(c_{m} \approx 10 \mathrm{nF} / \mathrm{mm}^{2}\right)$. Logo existe a relação entre a capacitância total e a capacitância específica, $C_{m}=c_{m} A$ ), onde $A$ é a área da superfície da membrana, que pode variar de $0,01 \mathrm{~mm}^{2}$ a $0,1 \mathrm{~mm}^{2}$, então a capacitância para todo o neurônio é tipicamente $0,1 \mathrm{nF}$ a $1 n F$. Como $d Q / d t$ é a corrente que entra na célula, então é possível determinar quanta corrente elétrica é necessária para mudar o potencial da membrana. A relação entre a taxa de variação do potencial e a corrente é dada por

$$
C_{m} \frac{d V}{d t}=\frac{d Q}{d t}
$$

A corrente também é determinada pela resistência da membrana. Se aplicarmos à célula uma corrente $I_{e}$, o potencial irá mudar do seu valor de repouso para uma quantidade $\Delta V$, que pela Lei de Ohm obedece a equação $\Delta V=I_{e} R_{m}$, onde $R_{m}$ é a resistência da membrana e ela pode variar em função da voltagem, então esse modelo é aceito para correntes e $\Delta V$ pequenas. A condutância da membrana é a inversa da resistência da membrana, mas a condutância pode ser escrita como $1 / r_{m}$, sendo que $r_{m}$ é a resistência específica da membrana e que $r_{m}=R_{m} A$, onde $A$ é a área da superfície da membrana. $O$ valor da resistência pode variar de célula para célula, seja por causa da variação da voltagem ou presença de canais iônicos.

Os íons estão sob ação de duas forças que os guiam através do canal iônico: difusão, que depende do gradiente de concentração, e da força elétrica, que depende da diferença de potencial $\Delta V$. Depois de certa quantidade de íons $K^{+}$tenha difundido para fora da célula, forma-se um potencial através da membrana em que a força elétrica se iguala à força química, ou seja, o movimento de saída (força química de $K^{+}$) do íon é igual ao movimento de entrada (força eletromotriz de $K^{+}$) (KANDEL; SCHWARTZ; JESSEL, 2000). É o chamado potencial de equilíbrio $E_{K}$. O potencial de equilíbrio para qualquer íons $i$ pode ser calculado pela equação de Nernst: 


$$
E_{i}=\frac{R T}{z F} \ln \frac{[i]_{e x t}}{[i]_{i n t}}
$$

onde $R$ é a constante dos gases, $T$ temperatura em Kelvin, $z$ a valência do íon, $F$ a constante de Faraday, e $[i]_{\text {ext }}$ e $[i]_{\text {int }}$ são as concentrações do íon fora e dentro da célula, respectivamente. Essa equação só se aplica quando somente um tipo de íon passa pelo canal. Mas como alguns canais não são totalmente seletivos, a equação de Goldman, que é somente uma adaptação da equação de Nernst, pode ser utilizada para calcular $E$ :

$$
E=\frac{R T}{F} \ln \frac{P_{K}\left[K^{+}\right]_{e x t}+P_{N a}\left[\mathrm{Na}^{+}\right]_{e x t}+P_{C l}\left[\mathrm{Cl}^{-}\right]_{i n t}}{P_{K}\left[K^{+}\right]_{i n t}+P_{N a}\left[N a^{+}\right]_{i n t}+P_{C l}\left[C l^{-}\right]_{e x t}}
$$

sendo que $P_{K}, P_{N a}$ e $P_{C l}$ são as permeabilidades relativas da membrana a cada espécie iônica. Em 1949, Alan Hodgkin e Bernard Katz foram os primeiros a aplicar sistematicamente a equação de Goldman na análise das variações do potencial de membrana, produzidas pela alteração das concentrações iônicas externas no axônio gigante da lula. Quando $V>E$, uma corrente positiva irá fluir para fora da célula, enquanto que se $V<E$ uma corrente positiva flui para dentro. Como as condutâncias de $\mathrm{Na}^{+}$e $\mathrm{Ca}^{2+}$ têm potencial de equilíbrio positivo, elas tendem a depolarizar um neurônio (o potencial é menos negativo); no caso de $K^{+}$, como o seu potencial de equilíbrio é negativo, então normalmente hiperpolariza a célula (o potencial é mais negativo).

A corrente total da membrana é determinada pela soma das correntes devidas aos diferentes tipos de canais presentes na membrana. Por convenção, é utilizada a corrente por unidade de área do neurônio, $i_{m}$. A corrente total é obtida multiplicando $i_{m}$ pela superfície celular $A$. Uma corrente, produzida por canais de algum íon $i$, deixará de existir quando $V=E_{i}$. Para muitos tipos de canais, a corrente aumenta ou diminui linearmente quando $V \neq E$. A diferença $V-E_{i}$ é chamada de força motriz e a corrente da membrana por unidade de área, para um tipo $i$ de canal é escrito como $g_{i}\left(V-E_{i}\right) \cdot g_{i}$ é a condutância por unidade de área. Se somarmos todas as correntes temos

$$
I_{m}=\sum_{j} g_{i}\left(V-E_{i}\right)
$$

Algumas características de uma célula nervosa dão complexidade à modelagem, como a presença de bombas de sódio-potássio, que afeta a condutância da membrana. No entanto, alguns desses fatores podem ser tratadas relativamente como constantes. Todas as contribuições, se forem independentes do tempo, podem ser agrupadas no termo $\bar{g}_{L}\left(V-E_{L}\right)$. $E_{L}$ é um parâmetro livre e pode ser ajustado para que o potencial de repouso do neurônio modelo coincida com a célula modelada. A condutância $\bar{g}_{L}$ é uma constante e também é ajustada conforme a condutância da membrana em repouso.

\section{Modelo matemático}

O artigo de Hodgkin e Huxley (1952) sintetiza e simplifica quase meio século de trabalhos com Eletrofisiologia (DAYAN; ABBOTT, 2001; KANDEL; SCHWARTZ; JESSEL, 2000). O que eles fizeram foi descrever o comportamento elétrico da membrana em termos de um circuito

SEPULVEDA, A. F. Modelo de Hodgkin-Huxley resolvido pelo método de Runge-Kutta. C.Q.D.- Revista Eletrônica Paulista de Matemática, Bauru, v. 11, p. 107-122, dez. 2017. Edição Iniciação Científica. 
elétrico equivalente (Figura 1). A corrente iônica é divida em corrente produzida pelo fluxo de íons sódio e potássio $\left(I_{N a}\right.$ e $I_{K}$, respectivamente), e uma pequena corrente $I_{L}$ produzida por $\mathrm{Cl}^{-}$ e outros íons. As condutâncias $g_{N a}$ e $g_{K}$ são em função do tempo e do potencial de membrana. $E_{N a}, E_{K}, E_{L}, C_{m}$ e $\bar{g}_{L}$ são tomadas como constantes. Pela Equação 1:

$$
I=c_{m} \frac{d V}{d t}+I_{m}
$$

onde $I$ é a densidade da corrente (influxo de corrente positiva), $I_{m}$ é a densidade de corrente iônica (influxo positivo de corrente), $V$ é a variação do potencial de membrana a partir do potencial de repouso (despolarização negativa), $c_{m}$ é a capacitância específica (ou a capacitância da membrana por unidade de área).

As correntes iônicas individuais são obtidas pelas relações

$$
\begin{aligned}
I_{N a} & =g_{N a}\left(E-E_{N a}\right)=g_{N a}\left(V-V_{N a}\right), \\
I_{K} & =g_{K}\left(E-E_{K}\right)=g_{K}\left(V-V_{K}\right), \\
I_{L} & =\bar{g}_{L}\left(E-E_{L}\right)=\bar{g}_{L}\left(V-V_{L}\right)
\end{aligned}
$$

onde

$$
\begin{gathered}
V=E-E_{r}, \\
V_{N a}=E_{N a}-E_{r}, \\
V_{K}=E_{K}-E_{r}, \\
V_{L}=E_{L}-E_{r},
\end{gathered}
$$

sendo que $E_{r}$ é o potencial de repouso e $V, V_{N a}, V_{K}$ e $V_{L}$ podem ser medidos como variação do potencial de membrana em relação ao potencial de membrana.

Estudando condutância do potássio:

$$
\begin{gathered}
g_{K}=\bar{g}_{K} n^{4}, \\
\frac{d n}{d t}=\alpha_{n}(1-n)-\beta_{n} n,
\end{gathered}
$$

onde $\bar{g}_{K}$ é uma constante com dimensões de condutância $/ \mathrm{cm}^{2}, \alpha_{n}$ e $\beta_{n}$ são constantes que dependem da voltagem, mas não do tempo, e têm dimensão de $[\text { tempo }]^{-1}, n$ representa a probabilidade de que algum íon $K^{+}$esteja dentro do canal. $\alpha_{n}$ é a taxa de transferência de íons potássio de fora para dentro da célula, já $\beta_{n}$ é a taxa de transferência de fora para dentro. Para o caso da condutância do sódio:

$$
\begin{gathered}
g_{N a}=m^{3} h \bar{g}_{N a}, \\
\frac{d m}{d t}=\alpha_{m}(1-m)-\beta_{m} m,
\end{gathered}
$$




$$
\frac{d h}{d t}=\alpha_{h}(1-h)-\beta_{h} h
$$

sendo $\bar{g}_{N a}$ uma constante e $\alpha$ e $\beta$ são funções de $V$ e não de $t$. Como Hodgkin e Huxley propõem, a condutância do sódio é proporcional ao número de sítios que são ocupados por três moléculas ativadoras e não são bloqueadas por alguma molécula inativadora. $m$ é a probabilidade de que alguma molécula ativadora esteja dentro do canal, enquanto que $1-m$ mostra a proporção das moléculas que estão fora. $h$ é a proporção de moléculas inativadoras no lado de fora e $1-h$ a proporção das que estão fora do canal.

A corrente total $I$ por unidade de área pode ser descrita pela soma das correntes iônicas:

$$
\begin{gathered}
I_{m}=I_{K}+I_{N a}+I_{L} \\
I_{m}=\bar{g}_{K} n^{4}\left(V-E_{K}\right)+\bar{g}_{N a} m^{3} h\left(V-E_{N a}\right)+\bar{g}_{L}\left(V-E_{L}\right) \\
I=c_{m} \frac{d V}{d t}+I_{m}
\end{gathered}
$$

O primeiro termo do lado direito pode ser resolvido como um modelo com uma única variável $V$ :

$$
c_{m} \frac{d V}{d t}=-i_{m}+\frac{I_{e}}{A}
$$

onde $I_{e}$ é a corrente injetada por um eletrodo. Lembrando a Equação 3:

$$
c_{m} \frac{d V}{d t}=-\bar{g}_{L}\left(V-E_{L}\right)+\frac{I_{e}}{A}
$$

Se multiplicarmos ambos os lados da equação acima pela resistência específica $r_{m}$, que nesse caso é dado por $r_{m}=1 / \bar{g}_{L}$, e como $\tau=r_{m} c_{m}$, temos:

$$
\tau \frac{d V}{d t}=E_{L}-V+R_{m} I_{e}
$$

Se integrarmos ambos os lados dessa equação obtemos:

$$
V(t)=E_{L}+R_{m} I_{e}+\left(V_{0}-E_{L}-R_{m} I_{e}\right) \exp (-t / \tau)
$$

onde $V_{0}$ é o potencial quando $t=t_{0}$. Os parâmetros $\alpha$ e $\beta$ dependem somente dos valores instantâneos dos potenciais de membrana. Hodgkin e Huxley verificaram experimentalmente o comportamento da condutância do respectivo íon quando o axônio é submetido a diferentes voltagens. As curvas obtidas, quando modeladas conforme os dados experimentais, respeitando as Equações 7, 9 e 10, devem ter como parâmetros

$$
\begin{gathered}
\alpha_{n}=0,01(V+55) /(1-\exp [-(V+10) / 10]), \\
\beta_{n}=0,125 \exp [-(V+65) / 80],
\end{gathered}
$$




$$
\begin{gathered}
\alpha_{m}=0,1(V+25) /(1-\exp [-(V+40) / 10]), \\
\beta_{m}=4 \exp [-(V+65) / 18], \\
\alpha_{h}=0,07 \exp [-(V+35) / 20] \\
\beta_{h}=1 /(1+\exp [(-V+35) / 10])
\end{gathered}
$$

\section{Métodos numéricos}

A Equação 10 é resolvida a partir da solução das equações diferenciais ordinárias 6, 8 e 9. Uma forma de resolver essas equações é pelo método de Runge-Kutta de Ordem Quatro. Generalizando, para uma equação diferencial ordinária (EDO) $y^{\prime}=f(t, y)$, definido no intervalo $a \leqslant t \leqslant b$, com valor inicial $y(a)=y_{0}$, o passo de integração será $\Delta t=(b-a) / N$, o método terá a forma

$$
y_{j+1}=y_{j}+\frac{1}{6}\left(k_{1}+2 k_{2}+2 k_{3}+k_{4}\right)
$$

para cada $j=0,1,2, \ldots, N$; e que

$$
\begin{aligned}
& k_{1}=\Delta t f\left(t_{j}, y_{j}\right), \\
& k_{2}=\Delta t f\left(t_{j}+\frac{1}{2}, y_{j}+\frac{1}{2} k_{1}\right), \\
& k_{3}=\Delta t f\left(t_{j}+\frac{1}{2}, y_{j}+\frac{1}{2} k_{2}\right), \\
& k_{4}=\Delta t f\left(t_{j+1}, y_{j}+k_{3}\right)
\end{aligned}
$$

Um ponto a favor na utilização do método de Runge-Kutta é o erro local do truncamento de alta ordem $\left(O\left(\Delta t^{4}\right)\right)$, ou seja, quanto menor o valor de $\Delta t$, menor será o valor do erro em cada passo. A solução da equação da corrente exige a discretização das EDO's.

\subsection{Discretização}

Seja definido o intervalo de tempo $t_{0} \leqslant t \leqslant t_{f}$ e $\Delta t_{n}$ o passo de integração, $V_{0}$ o potencial de membrana no instante $t=t_{0}$, a probabilidade de abertura de canal de potássio tem a forma $f(t, n)=n^{\prime}$ (Equação 6), com o valor inicial $n(0)$ no instante $t=0$. Respeitando os parâmetros $\alpha_{n}$ e $\beta_{n}$ temos para $j=0,1,2, \ldots N$ :

$$
\begin{aligned}
& k_{1, n, j}=\Delta t\left[\alpha_{n, j}\left(1-n_{j}\right)-\beta_{n, j} n_{j}\right] \\
& k_{2, n, j}=\Delta t\left[\alpha_{n, j}\left(1-n_{j}+0.5 k_{1, n, j}\right)-\beta_{n, j}\left(n_{j}+0.5 k_{1, n, j}\right)\right] \\
& k_{3, n, j}=\Delta t\left[\alpha_{n, j}\left(1-n_{j}+0.5 k_{2, n, j}\right)-\beta_{n, j}\left(n_{j}+0.5 k_{2, n, j}\right)\right] \\
& k_{4, n, j}=\Delta t\left[\alpha_{n, j}\left(1-n_{j}++k_{3, n, j}\right)-\beta_{n, j} n_{j}+k_{3, n, j}\right]
\end{aligned}
$$


onde

$$
\begin{aligned}
& \alpha_{n, j}=0,01\left(V_{j}+55\right) /\left(1-\exp \left(-\left(V_{j}+10\right) / 10\right)\right) \mathrm{e} \\
& \beta_{n, j}=0,125 \exp \left(-\left(V_{j}+65\right) / 80\right)
\end{aligned}
$$

se resulta em

$$
\begin{aligned}
n_{j+1} & =n_{j}+\frac{1}{6}\left(k_{1, n, j}+2 k_{2, n, j}+2 k_{3, n, j}+k_{4, n, j}\right) \\
t_{j+1} & =t_{j}+\Delta t
\end{aligned}
$$

No caso da probabilidade de $m$ temos $f(t, m)=m^{\prime}$ e $g(t, h)=h^{\prime}$, onde existem os valores iniciais $m_{0}$ e $h_{0}$ e, com parâmetros 13-16, temos:

$$
\begin{aligned}
& k_{1, m, j}=\Delta t\left[\alpha_{m, j}\left(1-m_{j}\right)-\beta_{m, j} m_{j}\right] \\
& k_{2, m, j}=\Delta t\left[\alpha_{m, j}\left(1-m_{j}+0.5 k_{1, m, j}\right)-\beta_{m, j}\left(m_{j}+0.5 k_{1, m, j}\right)\right] \\
& k_{3, m, j}=\Delta t\left[\alpha_{m, j}\left(1-m_{j}+0.5 k_{2, m, j}\right)-\beta_{m, j}\left(m_{j}+0.5 k_{2, m, j}\right)\right] \\
& k_{4, m, j}=\Delta t\left[\alpha_{m, j}\left(1-m_{j}+k_{3, m, j}\right)-\beta_{m, j}\left(m_{j}+k_{3, m, j}\right)\right]
\end{aligned}
$$

e para $h$

$$
\begin{aligned}
& k_{1, h, j}=\Delta t\left[\alpha_{h, j}\left(1-h_{j}\right)-\beta_{h, j} h_{j}\right] \\
& k_{2, h, j}=\Delta t\left[\alpha_{h, j}\left(1-h_{j}+0.5 k_{1, m, j}\right)-\beta_{h, j}\left(h_{j}+0.5 k_{1, m, j}\right)\right] \\
& k_{3, h, j}=\Delta t\left[\alpha_{h, j}\left(1-h_{j}+0.5 k_{2, m, j}\right)-\beta_{h, j}\left(h_{j}+0.5 k_{2, m, j}\right)\right] \\
& k_{4, h, j}=\Delta t\left[\alpha_{h, j}\left(1-h_{j}+k_{3, m, j}\right)-\beta_{h, j}\left(h_{j}+k_{3, m, j}\right)\right]
\end{aligned}
$$

sendo

$$
\begin{aligned}
\alpha_{m, j} & =0,1\left(V_{j}+40\right) /\left(1-\exp \left(-\left(V_{j}+25\right) / 10\right)\right) \\
\beta_{m, j} & =4 \exp \left(-\left(V_{j}+65\right) / 18\right) \\
\alpha_{h, j} & =0,07 \exp \left(-\left(V_{j}+65\right) / 20\right) \mathrm{e} \\
\beta_{h, j} & =1 /\left(1+\exp \left(-\left(V_{j}+35\right) / 10\right)\right)
\end{aligned}
$$

Logo,

$$
\begin{aligned}
m_{j+1} & =m_{j}+\frac{1}{6}\left(k_{1, m, j}+2 k_{2, m, j}+2 k_{3, m, j}+k_{4, m, j}\right) \mathrm{e} \\
h_{j+1} & =h_{j}+\frac{1}{6}\left(k_{1, h, j}+2 k_{2, h, j}+2 k_{3, h, j}+k_{4, h, j}\right)
\end{aligned}
$$

Cada $V_{j}$ é resolvido pela Equação 10, também por Runge-Kutta: 


$$
\begin{aligned}
& k_{1, j}=\Delta t\left[\left(1 / C_{m}\right)\left(-i_{m}+I_{e} / A\right)\right] \\
& k_{2, j}=\Delta t\left[\left(1 / C_{m}\right)\left(-i_{m}+I_{e} / A+0.5 k_{1, j}\right)\right] \\
& k_{3, j}=\Delta t\left[\left(1 / C_{m}\right)\left(-i_{m}+I_{e} / A+0.5 k_{2, j}\right)\right] \\
& k_{4, j}=\Delta t\left[\left(1 / C_{m}\right)\left(-i_{m}+I_{e} / A+k_{3, j}\right)\right]
\end{aligned}
$$

Lembrando que:

$$
\begin{aligned}
i_{m} & =I_{K}+I_{N a}+I_{L} \\
I_{K} & =g_{K} n_{j}^{4}\left(V_{j}-E_{K}\right) \\
I_{N a} & =g_{N a} m_{j}^{3} h_{j}\left(V_{j}-E_{N a}\right) \\
I_{L} & =g_{L}\left(V-E_{L}\right)
\end{aligned}
$$

onde $E_{N a}$ e $E_{K}$ são obtidos pela Equação 2. Temos então

$$
V_{j+1}=V_{j}+\frac{1}{6}\left(k_{1, j}+2 k_{2, j}+2 k_{3, j}+k_{4, j}\right)
$$

\subsection{Análise do erro e convergência numérica}

A aplicação de um método numérico na solução de uma EDO pode provocar alguma variação em torno do valor verdadeiro, ou seja, existe um erro no valor resultante $O\left(\Delta t^{\mu}\right)$. Como o erro $\varepsilon$ é tal que

$$
\varepsilon_{\mu}=|f-y| \approx C(t) \Delta t^{p}
$$

onde $f$ é o valor verdadeiro de uma equação e $y$ é o valor obtido pelo método numérico. $\mu$ é um número inteiro tal que o passo de integração seja

$$
\Delta t_{\mu}=\frac{b-a}{4^{\mu}}
$$

dentro de um intervalo $a \leqslant t \leqslant b$. Devemos avaliar o erro nos cálculos de $m, n$ e $h$. Para isso, verifica-se para que valores de $\mu$ as soluções numéricas se tornam cada vez mais próximas, logo já eliminam-se os casos em que essas soluções estão mais distantes do valor verdadeiro.

Para um instante $t$ qualquer:

$$
\varepsilon\left(t, \Delta t_{\mu}\right)=\left|f\left(t, \Delta t_{\mu}\right)-y(t)\right|
$$

E usando $\Delta t / 4$ :

$$
\frac{|\varepsilon(t, \Delta t)|}{|\varepsilon(t, \Delta t / 4)|}=\frac{|f(t, \Delta t)-y(t)|}{|f(t, \Delta t / 4)-y(t)|} \approx \frac{C(t) \Delta t^{\mu}}{C(t)(\Delta t / 4)^{\mu}}=4^{\mu}
$$

Se calcularmos a razão $r=\varepsilon\left(t, \Delta t_{\mu}\right) / \varepsilon\left(t, \Delta t_{\mu+1}\right)$, para todos os $\mu$, todos $r$ devem ser aproximadamente iguais, se o algoritmo for corretamente implementado. Se tomarmos

$$
p=\log _{4} r
$$


os valores de $p$ irão convergir.

\section{Resultados}

Existem muitos exemplos de implementação do modelo de Hodgkin-Huxley amplamente disponíveis, geralmente em Matlab e resolvidos pelo método de Euler. A vantagem de ser implementado por esse método é o relativo menor custo de tempo para implementar no computador, porém é menos exato que o método de Runge-Kutta. O modelo foi implementado através da linguagem Python (a rotina está implementado em https://github.com/And-Sepulveda/). Para determinar para que valores de $\mu$ a solução numérica se aproxima da solução verdadeira, o método foi implementado para cada $\mu$.

Conforme o valor de $\mu$ aumenta, a solução numérica mais se aproxima do valor verdadeiro, porém quanto maior for esse valor, mais memória do computador será utilizada, então mais tempo necessário para resolver o problema. A Figura 2 mostra o comportamento do potencial da membrana depois do disparo do potencial de ação, quando o potencial de repouso é $V_{\text {rep }}=-65$ $m V$.
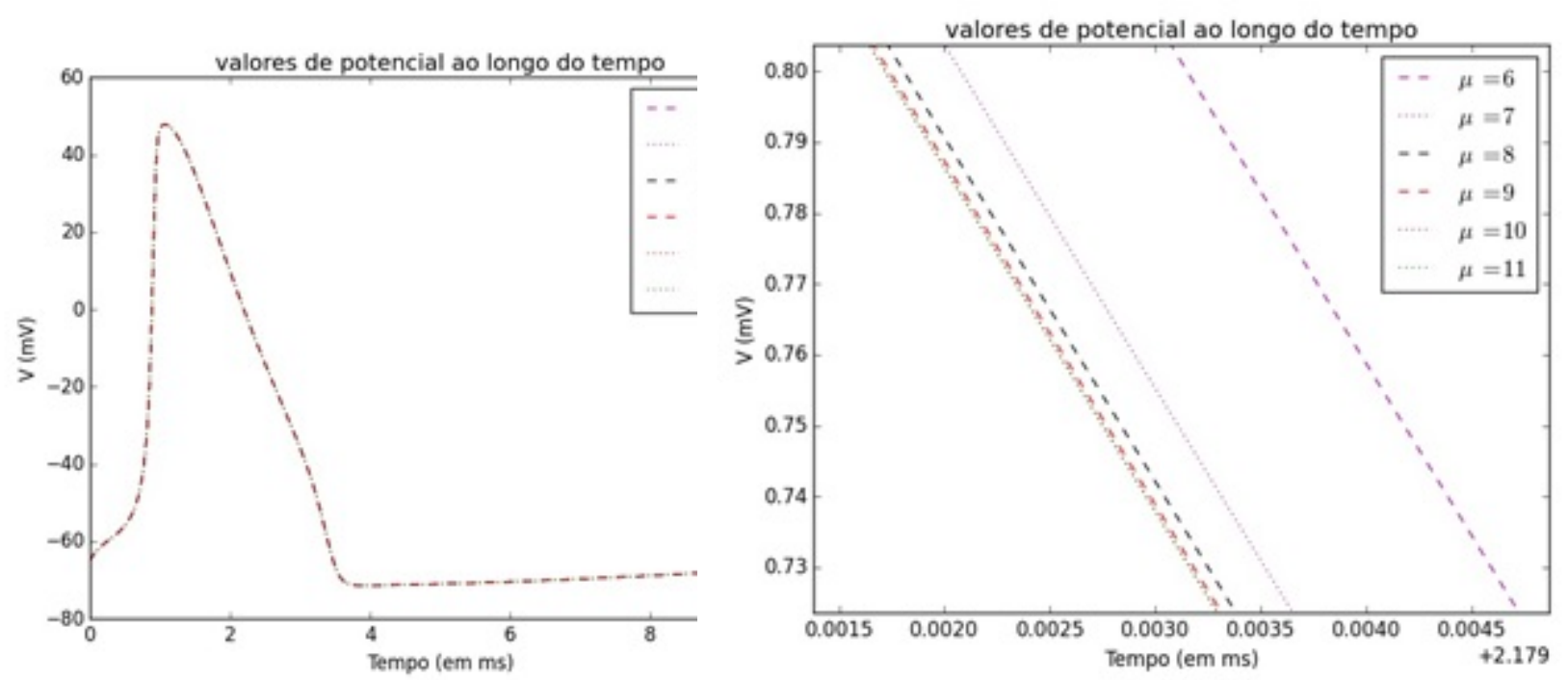

Figura 2: Potencial de membrana varia com o tempo logo depois do disparo do potencial de ação. O potencial máximo, quando a membrana é despolarizada, ocorre quando o potencial é igual ao potencial de equilíbrio de $\mathrm{Na}^{+}\left(E_{N a} \approx 54 \mathrm{mV}\right)$. Expandindo o gráfico (à direita), observa-se a tendência de aproximação da solução numérica com a solução exata.

O potencial da membrana depende da abertura e fechamento dos canais. Quando o potencial da membrana alcança o valor aproximado de $-50 \mathrm{mV}$, os canais de sódio são abertos, ou seja, o valor de $m$, que era quase zero passa a tender a 1 , isto é, quase todos os canais estão abertos para passagem de íons sódio (Figura 3). 

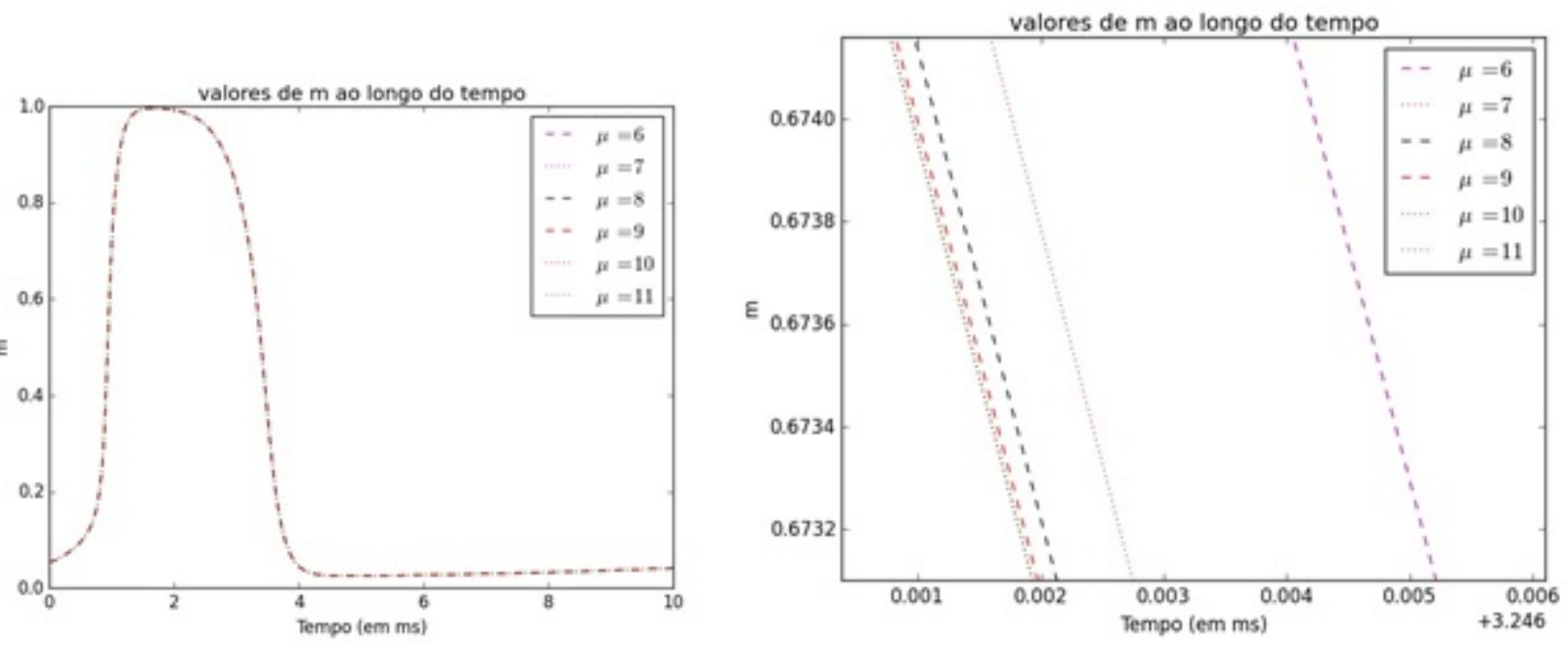

Figura 3: A probabilidade $m$ de abertura de canais de $N a^{+}$, onde o valor inicial $m_{0} \approx 0,05$ quando a membrana está com potencial de repouso. Quando o gráfico é expandido, observa-se que a solução numérica tende à solução exata quanto maior for $\mu$.

O valor de $h$ expressa a inativação da condutância do canais de $\mathrm{Na}^{+}$. Conforme a membrana despolariza, o valor de $h$ diminui, os canais de sódio são abertos e então há influxo de $\mathrm{Na}^{+}$, o que aumenta o valor de $V$ (Figura 4).
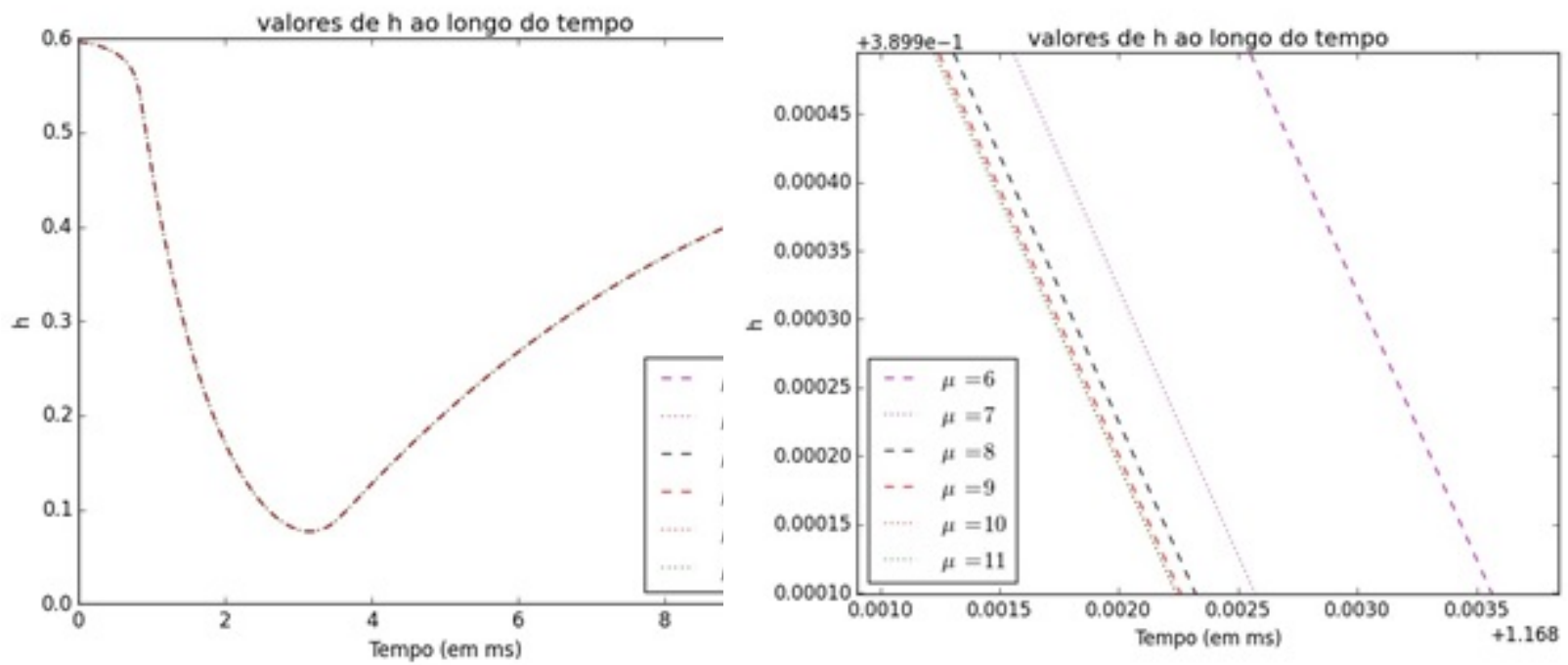

Figura 4: A probabilidade $h$ de inativação de canais de $N a^{+}$, com valor inicial $h_{0} \approx 0,06$ quando o potencial de repouso $V_{\text {rep }}=-65 \mathrm{mV}$.

O aumento do potencial faz com que a condutância ao sódio seja inativado ( $h$ tende a zero), diminuindo o influxo de $\mathrm{Na}^{+}$. A despolarização da membrana ativa também a condutância ao $K^{+}$, aumentando o valor de $n$ (Figura 5). 

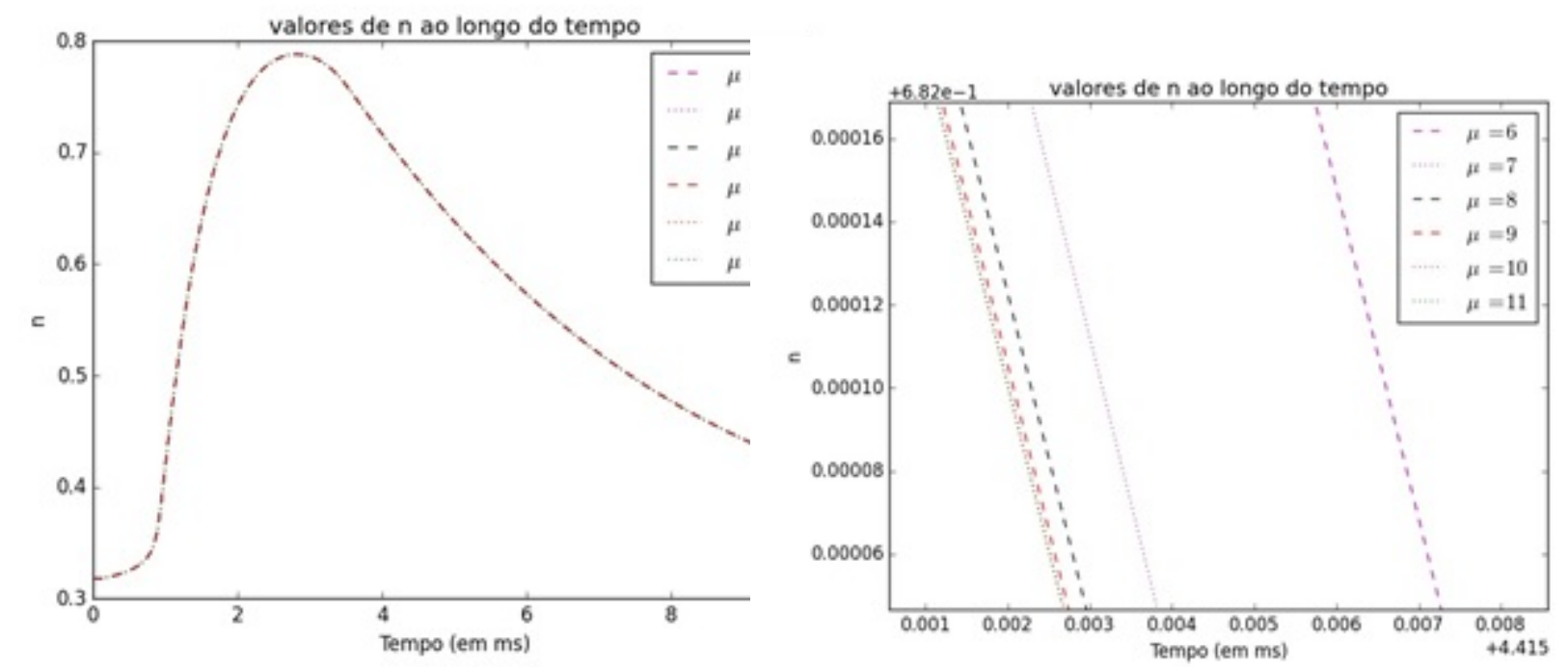

Figura 5: Probabilidade $n$ de ativação de canais de $K^{+}$ao longo do tempo. No potencial de repouso, no instante inicial, $n_{0} \approx 0.35$. Novamente, a solução numérica se aproxima do valor real quanto maior for o valor de $\mu$.

O grande influxo de sódio produz um forte e rápido influxo de corrente (Figura 6), o que causa o rápido aumento do potencial até o valor do potencial de equilíbrio de $\mathrm{Na}^{+}$.

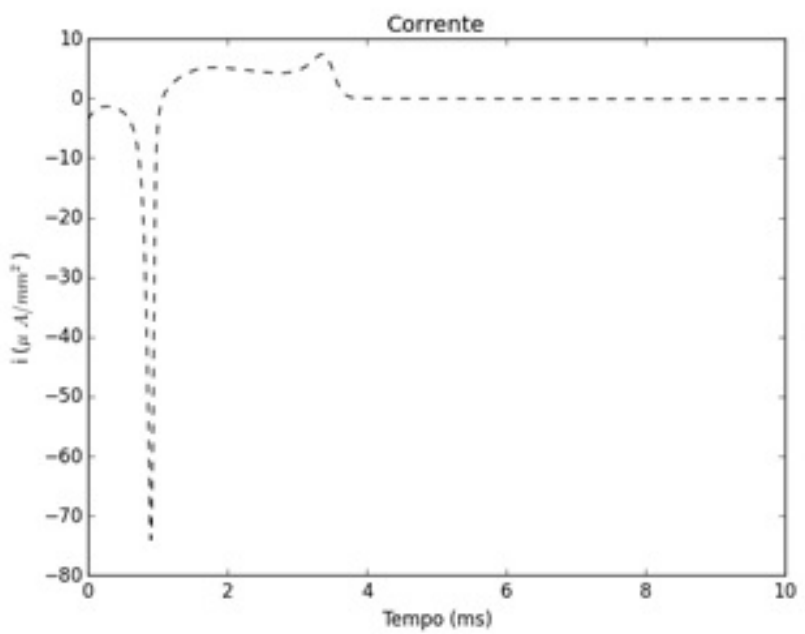

Figura 6: Densidade de corrente (em $\mu \mathrm{A} / \mathrm{mm}^{2}$ ) ao longo do intervalo de tempo

Para facilitar a visualização da dinâmica do modelo de Hodgkin-Huxley, a Figura 7 resume o papel dos canais para a propagação do potencial de ação ao longo do axônio. 


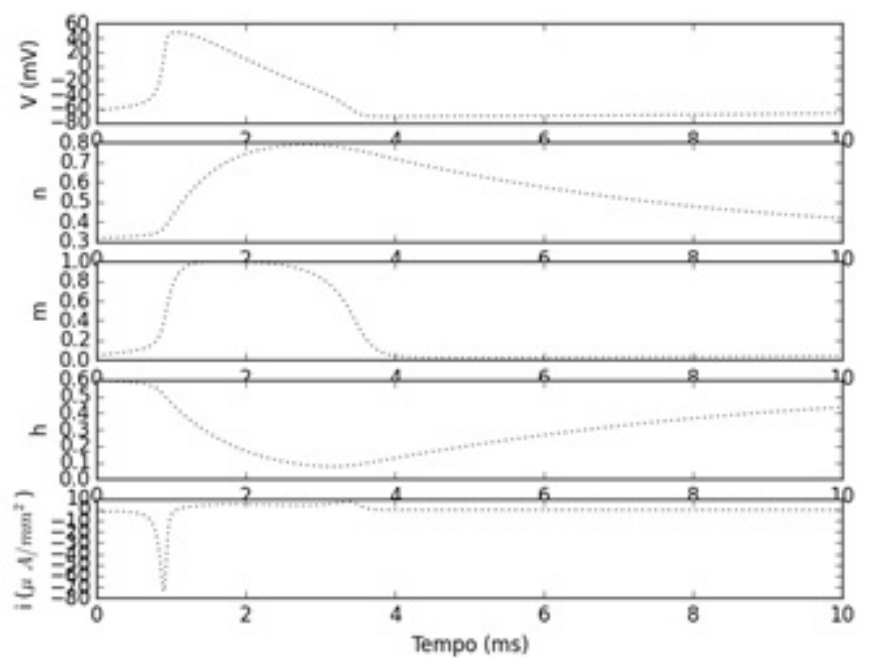

Figura 7: Quando uma célula sofre um estímulo, há despolarização da membrana, provocando a abertura dos canais de sódio. Essa abertura aumenta o influxo de $\mathrm{Na}^{+}$(e consequentemente da corrente), o que despolariza ainda mais a membrana. Esse incremento de despolarização diminui a probabilidade $h$, ou seja, a condutância ao sódio diminui (os canais de $\mathrm{Na}^{+}$inativam-se), que em consequência o influxo de sódio é inibido. A despolarização também aumenta a abertura de canais de $K^{+}$, que hiperpolariza a membrana, voltando ao valor de potencial de repouso.

Para descobrir os valores exatos, e comparar com as soluções numéricas obtidas pelo método de Runge-Kutta, devemos integrar as equações 6, 8 e 9. Primeiro integrando a Equação 7 temos

$$
n=n_{\infty}-\left(n_{\infty}-n_{0}\right) \exp \left(-t / \tau_{n}\right)
$$

onde

$$
\begin{aligned}
n_{\infty} & =\frac{\alpha_{n}(V)}{\alpha_{n}(V)+\beta_{n}(V)} \\
\tau_{n} & =\frac{1}{\alpha_{n}(V)+\beta_{n}(V)}
\end{aligned}
$$

Resolvendo a Equação 9:

$$
m=m_{\infty}-\left(m_{\infty}-m_{0}\right) \exp \left(-t / \tau_{m}\right)
$$

onde

$$
\begin{aligned}
m_{\infty} & =\frac{\alpha_{m}(V)}{\alpha_{m}(V)+\beta_{m}(V)} \\
\tau_{m} & =\frac{1}{\alpha_{m}(V)+\beta_{m}(V)}
\end{aligned}
$$


E a Equação 10:

$$
h=h_{\infty}-\left(h_{\infty}-n_{0}\right) \exp \left(-t / \tau_{h}\right)
$$

onde

$$
\begin{aligned}
h_{\infty} & =\frac{\alpha_{h}(V)}{\alpha_{h}(V)+\beta_{h}(V)} \\
\tau_{h} & =\frac{1}{\alpha_{n}(V)+\beta_{h}(V)}
\end{aligned}
$$

sendo que $n_{0}, m_{0}$ e $h_{0}$ são as probabilidades de $n, m$ e $h$, respectivamente, quando a membrana está em potencial de repouso. $n_{\infty}, m_{\infty}$ e $h_{\infty}$ descrevem a estabilidade dos canais. $\tau_{n}, \tau_{m}$ e $\tau_{h}$ são constantes de tempo em $m s$. Em todos os casos, eles dependem do valor do potencial. Como existem vários exemplos de EDO's de difícil solução, foram optados os valores de potencial que são mais próximos da solução exata, que nesse caso é quando $\mu=11$. As soluções das equações acima para o intervalo de tempo $0 \leqslant t \leqslant 10 \mathrm{~ms}$ e $\mu=11$ foram obtidas por implementação em Python.

Como $h_{\infty}$ corresponde a uma variável de inativação, ele tem um comportamento oposto de $n_{\infty}$ e $m_{\infty}$. Quando o potencial assumes valores mais negativos (isto é, a membrana está hiperpolarizada), $h_{\infty}$ tende a 1 , e quando a membrana está despolarizada, $h_{\infty}$ se torna nula (Figura 8).
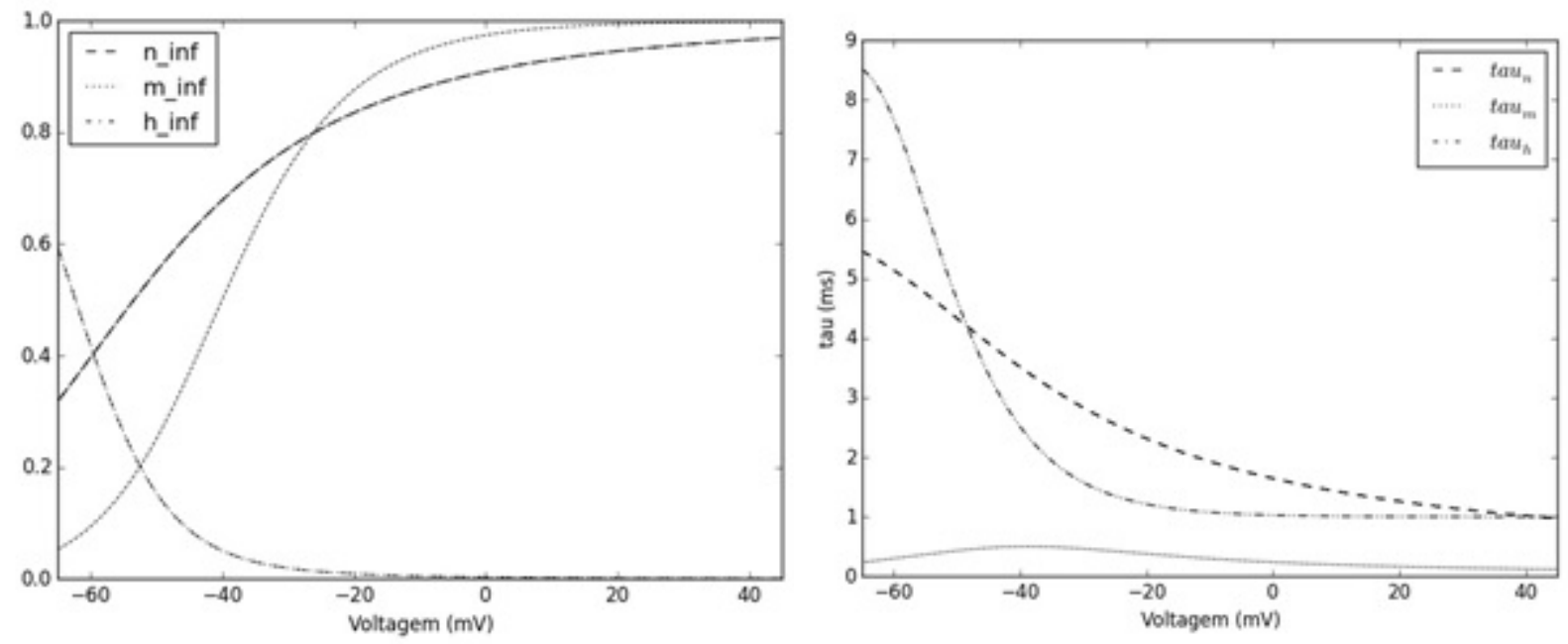

Figura 8: O gráfico à esquerda mostra os valores de $n_{\infty}, m_{\infty}$ e $h_{\infty}$, que são os níveis de estabilidade de ativação e inativação de condutância de $\mathrm{Na}^{+}$e ativação de condutância de $\mathrm{K}^{+}$. O gráfico à direita mostra as constantes de tempo $\tau_{n}, \tau_{m}$ e $\tau_{h}$, que controlam as taxas em que os níveis de estabilidade são aproximadas.

Para análise da convergência da solução numérica à solução exata, foi implementada a Equação 18 para os valores de $6 \leqslant \mu \leqslant 11$. Com o resultado obtido, foram montadas tabelas de convergência. Na comparação dos valores de $n$ da solução numérica com a solução exata pela Equação19: 
Tabela 1: Análise de convergência de $n$

\begin{tabular}{c|c|c|c}
\hline$\mu$ & $e\left(t, \Delta t_{m}\right)$ & $r=\frac{e\left(t, \Delta t_{m}\right)}{e\left(t, \Delta t_{m+1}\right)}$ & $\log _{2} r$ \\
\hline 6 & $1,68 \mathrm{E}-05$ & 15,8 & 1,99 \\
7 & $1,06 \mathrm{E}-06$ & 16,0 & 2,00 \\
8 & $6,65 \mathrm{E}-08$ & 16,1 & 2,00 \\
9 & $4,13 \mathrm{E}-09$ & 17,8 & 2,08 \\
10 & $2,31 \mathrm{E}-10$ & 19,0 & 2,12 \\
\hline
\end{tabular}

Confirmando o que foi observado graficamente, para valores mais altos de $\mu$ a solução numérica se aproxima de solução exata e existe convergência $p \approx 2,00$. No caso de $m$ :

Tabela 2: Análise de convergência de $m$

\begin{tabular}{c|c|c|c}
\hline$\mu$ & $e\left(t, \Delta t_{m}\right)$ & $r=\frac{e\left(t, \Delta t_{m}\right)}{e\left(t, \Delta t_{m+1}\right)}$ & $\log _{2} r$ \\
\hline 6 & $1,53 \mathrm{E}-05$ & 0,45 & $-0,57$ \\
7 & $3,38 \mathrm{E}-05$ & 3,28 & 0,86 \\
8 & $1,03 \mathrm{E}-05$ & 3,98 & 1,00 \\
9 & $2,59 \mathrm{E}-06$ & 4,78 & 1,13 \\
10 & $5,41 \mathrm{E}-07$ & 24,8 & 2,32 \\
\hline
\end{tabular}

E no caso de $h$ :

Tabela 3: Análise de convergência de $h$

\begin{tabular}{c|c|c|c}
\hline$\mu$ & $e\left(t, \Delta t_{m}\right)$ & $r=\frac{e\left(t, \Delta t_{m}\right)}{e\left(t, \Delta t_{m+1}\right)}$ & $\log _{2} r$ \\
\hline 6 & $2,46 \mathrm{E}-05$ & 15,8 & 1,99 \\
7 & $1,55 \mathrm{E}-06$ & 16,0 & 2,00 \\
8 & $9,72 \mathrm{E}-08$ & 16,1 & 2,00 \\
9 & $6,04 \mathrm{E}-09$ & 17,8 & 2,08 \\
10 & $3,39 \mathrm{E}-10$ & 19,0 & 2,12 \\
\hline
\end{tabular}

Se comparados todos os pontos entre as duas soluções, serão obtidos erros pequenos, cada vez menores com valores maiores de $\mu$. Obviamente, pretende-se obter valores exatos, mas se não for possível resolver uma EDO, o método de Runge-Kutta pode ser suficiente para termos soluções mais próximas possível.

\section{Conclusão}

Escolhendo implementar o modelo pelo método de Runge-Kutta, as soluções numéricas são mais próximas da solução exata, em comparação com outros métodos, como o método de Euler. A desvantagem de trabalhar por esse método é o custo computacional da implementação.

O modelo de Hodgkin-Huxley é um dos modelos biológicos mais poderosos. A partir de conceitos físicos clássicos do Eletromagnetismo e experimentos relativamente simples, iniciaram uma era de trabalhos envolvendo Neurociência Computacional. 


\section{Referências bibliográficas}

BURDEN, R. L.; FAIRES, J. D. Initial-value problems for ordinary differential equations. In: . Numerical analysis. 8. ed. Belmont: Thomson Brooks/Cole, 2005. p. 278-280.

DAYAN, P.; ABBOTT, L. F. Model neurons I: neuroelectronics. In: Theoretical neuroscience: computational and mathematical. Cambridge. MIT Press, 2001. p. 154-194.

GERSTNER, W.; KISTLER, W. Detailed neuron models. In: Spiking neuron models: single neurons, populations, plasticity. Cambridge: Cambridge University Press, 2002. p. 31-67.

HODGKIN, A. L.; HUXLEY, A. F. A quantitative description of membrane current and its application to conduction and excitation in nerve. J. Physiol., v. 117, n. 4, p. 500-544, 1952.

KANDEL, E. R.; SCHWARTZ, J. H.; JESSEL, T. M. Membrane potencial. In: Principles of Neural Science. 4. ed. New York: McGraw-Hill, 2000. p. 125-139.

Artigo recebido em maio 2017 e aceito em nov. 2017. 